S. Kwok, M. Dopita, and R. Sutherland, eds.

\title{
Dust Features in Carbon-rich Planetary Nebulae
}

\author{
Albertina Pei \\ Dept. Physics and Astronomy, University of Calgary \\ Kevin Volk \\ Dept. Physics and Astronomy, University of Calgary
}

\section{Introduction}

Carbon-rich AGB stars, protoplanetary nebulae (PPNe), and PNe show a variety of dust features attributed to carbon-based compounds. Of particular interest are the three dust features peaking near 21,26 and $33 \mu \mathrm{m}$. The 26 and 33 $\mu \mathrm{m}$ features (collectively the " $30 \mu \mathrm{m}$ feature"; see Volk et al. 2002) are always observed together. The $21 \mu \mathrm{m}$ feature is weak in the AGB phase and becomes more prominent in the PPN phase, which implies carrier evolution. We wish to see how this evolution continues into the PN phase from detailed modelling of the infrared spectra of PNe.

The computer models were created in a two-component system. The ionized region is modelled by the photoionization code by G. Ferland (CLOUDY 90). Any neutral dust envelope is modelled with the radiative transfer program by C. Leung (DUSTCD). The final model spectrum is a combination of these results. The output model spectra are compared to data from the Infrared Space Observatory (ISO) and with the $\mathrm{H} \beta$ flux, optical line diagnostic ratios, the observed ionization front radius, and the stellar $\mathrm{V}$ magnitude. We assume that amorphous carbon grains produce the underlying continuum, and empirically add on features with strengths normalized to the underlying continuum.

\section{Results}

Our model geometry for IC 418 was adopted from Meixner et al (1996). There is a thin, high density shell and an external inverse-square density profile with a density jump of 4 . The distance to the nebula is taken to be $1.1 \mathrm{kpc}$. The central star is modelled with a $41,000 \mathrm{~K} \mathrm{Kurucz}$ atmosphere. Elemental abundances are taken from Henry, Kwitter \& Bates (2000).

We found discrepancies for the $11.3 \mu \mathrm{m}$ feature as shown in the Figure. The feature as observed in IC 418 is wider (FWHM $2.13 \mu \mathrm{m}$ ) than what is observed in the precursor AGB stars (FWHM $1.58 \mu \mathrm{m}$ ). The dust grains responsible for this feature seem to be changing in the post-AGB evolution. IC 418 shows a weak $21 \mu \mathrm{m}$ feature, which had not been realized previously. The strengths were found to be $0.2,1.05$, and 1.40 for the 21,26 , and $33 \mu \mathrm{m}$ features.

NGC 7027 is a non-spherical PN that was modeled using the average of three calculations using the semi-minor, semi-major, and average radius of the 

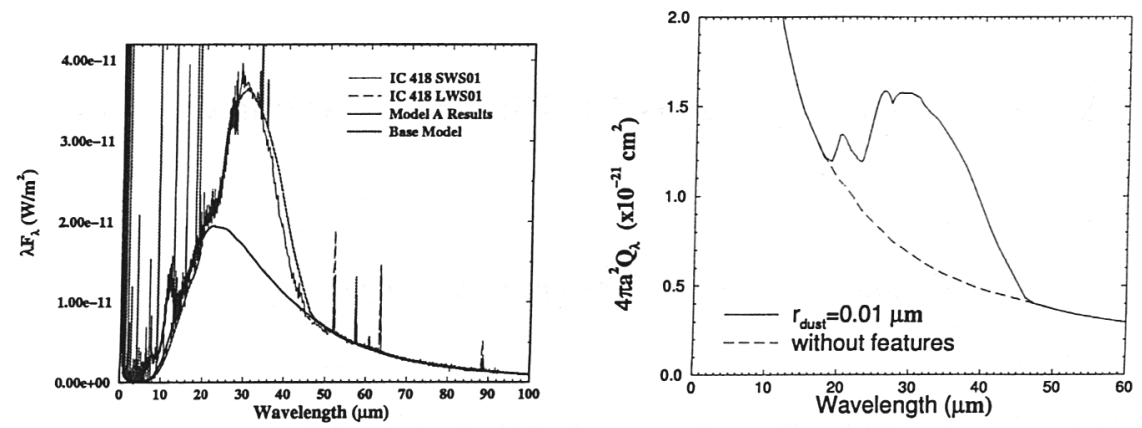

Figure 1. The model for IC 418: spectral fit and opacity function.

$\mathrm{PN}$. The ionized region is a thin, constant density region. The neutral region contains a high-density region at the inner edge, a density drop of a factor of 100 , and an inverse-square wind region. The distance is taken to be $0.93 \mathrm{kpc}$. The central star is modelled as a $198,000 \mathrm{~K}$ blackbody; elemental abundances are taken from Salas et al (2001). The strengths were found to be $<0.02,0.2$, and 0.3 for the 21,26 , and $33 \mu \mathrm{m}$ features; these low values may indicate grain destruction.
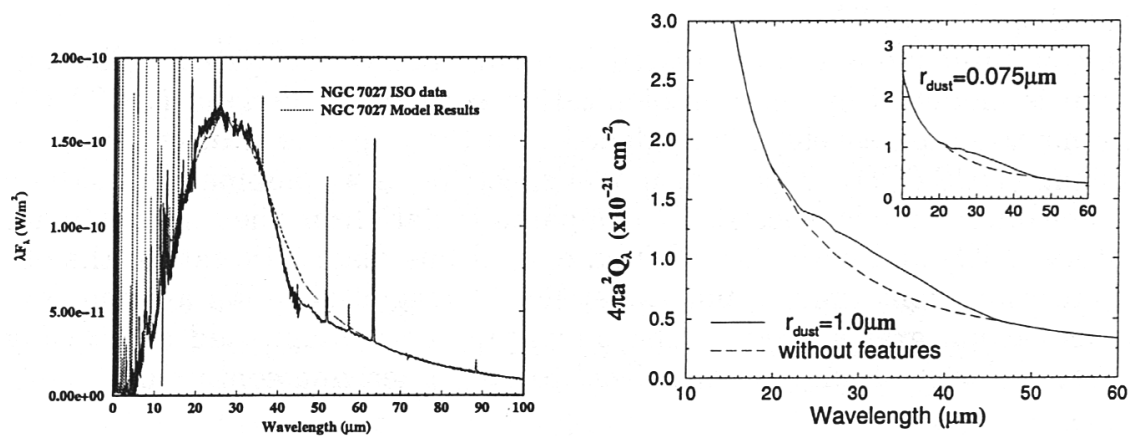

Figure 2. The model for NGC 7027: spectral fit and opacity function.

The PN models provide a good fit to our main observational constraints. The 26/33 $\mu \mathrm{m}$ feature profiles derived from PPNe (Volk et al. 2002) match the features in these PN, so these features show no evolution beyond the PPN phase.

\section{References}

Henry, R. B. C., Kwitter, K. B., \& Bates, J. A. 2000, ApJ, 531, 928

Meixner, M., et al. 1996, A\&A, 313, 234

Salas, J. B., et al. 2001, A\&A, 367, 949

Volk, K., Kwok, S., Hrivnak, B.J., \& Szczerba, R. 2002, ApJ, 567, 412 